\title{
THE EQUIVALENCE AMONG THE MODIFIED MANN-ISHIKAWA AND NOOR ITERATIONS FOR UNIFORMLY L-LIPSCHITZIAN MAPPINGS IN BANACH SPACES
}

\author{
ZHIQUN XUE
}

Abstract. In this paper, the equivalence of the convergence among Mann-Ishikawa and Noor iterations is obtained for uniformly L-Lipschitzian mappings in real Banach spaces. Our results extend and improve the corresponding results in Chang [3] and Ofoedu [4].

Mathematics subject classification (2010): 47H10, 47H09, 46B20..

Keywords and phrases: uniformly L-Lipschitzian mapping, the modified Mann and Ishikawa iterations, the modified Noor iteration, real Banach space..

\section{REFERENCES}

[1] K. Gobel AND W. A. KIRK, A fixed point theorem for asymptotically nonexpansive mappings, Proc.Amer.Math.Soc., 35, 1 (1972), 171-174.

[2] J. SchU, Iterative construction of fixed points of asymptotically nonexpansive mappings, J. Math. Anal. Appl., 158 (1991), 407-413.

[3] S. S. ChAng, Y. J. Cho, J. K. Kim, Some results for uniformly L-Lipschitzian mappings in Banach spaces, Appied Mathematics Letters., 22, 1 (2009), 121-125.

[4] E. U. OFOEDU, Strong convergence theorem for uniformly L-Lipschitzian asymptotically pseudocontractive mapping in real Banach space, J. Math. Anal. Appl, 321 (2006), 722-728.

[5] X. Weng, Fixed point iteration for local strictly pseudocontractive mapping, Proc. Amer. Math. Soc, 113 (1991), 727-731.

[6] M. A. Noor, New approximation schemes for general variational inequalities, J. Math. Anal. Appl., 251 (2000), 217-229.

[7] M. A. NooR, Three-step iterative algorithms for multivalued quasi variational inclusions, J. Math. Anal. Appl., 255 (2001).

[8] M. A. Noor, T. M. Rassias, Z. Y. HUANG, Three-step iterations for nonlinear accretive operator equations, J. Math. Anal. Appl., 274 (2002), 59-68.

[9] M. A. Noor, Some developments in general variational inequalities, Appl. Math. Computation, 152 (2004), 199-277.

[10] R. ARIF, Modified Noor iterations for nonlinear equations in Banach spaces, Appl. Math. Computation, 182 (2006), 589-595.

[11] M. A. NOOR, Z. Y. HUANG, Three-step methods for nonexpansive mappings and variational inequalities, Appl. Math. Computation, 187 (2007), 680-685.

[12] Xue Z. Q, FAn R. Q, Some comments on Noor's iterations in Banach spaces, Appl. Math. Computation, 206, 1 (2008), 12-15. 